\title{
STING expression in monocyte-derived macrophages is associated with the progression of liver inflammation and fibrosis in patients with nonalcoholic fatty liver disease
}

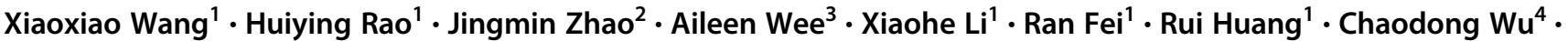 \\ Feng Liu $^{1} \cdot$ Lai Wei $^{1,5}$
}

Received: 6 May 2019 / Revised: 27 September 2019 / Accepted: 17 October 2019 / Published online: 19 November 2019

(c) The Author(s), under exclusive licence to United States and Canadian Academy of Pathology 2019

\begin{abstract}
The stimulator of interferon genes (STING) in macrophages plays a crucial role in nonalcoholic fatty liver disease (NAFLD) progression. However, there is a lack of evidence from large samples of patients to validate a deleterious role for STING in NAFLD. Moreover, sources of STING-expressing cells that are related to NAFLD remain to be definitively characterized. To investigate STING expression and explore its correlation with NAFLD progression in human subjects, our study involved liver samples from 98 NAFLD subjects and 8 controls. STING and p-TBK1 expression in nonparenchymal liver cells was analyzed and correlated with NAFLD pathological features. Numbers of $\mathrm{STING}^{+}$cells were increased in livers from nonalcoholic steatohepatitis (NASH) patients compared with controls, especially in the liver portal tract of NASH patients with fibrosis $(p<0.05)$. Moreover, numbers of $\mathrm{STING}^{+}$cells in livers of NASH patients were increased with aggravation of inflammation grade and fibrosis stage $(p<0.05)$. STING was mainly expressed in macrophages, including monocyte-derived macrophages $\left(\mathrm{CCR} 2^{+}, \mathrm{S} 100 \mathrm{~A} 9^{+}\right)$, Kupffer cells $\left(\mathrm{CD} 68^{+}\right)$and $\mathrm{CD} 163^{+}$macrophages. Compared with controls, numbers of $\mathrm{STING}^{+} / \mathrm{CCR}^{+}$and $\mathrm{STING}^{+} / \mathrm{S} 100 \mathrm{~A}^{+}$cells were significantly increased in livers from NASH patients with fibrosis and positively correlated with liver inflammation grade and fibrosis stage $(p<0.05)$. However, numbers of STING ${ }^{+} / \mathrm{CD} 68^{+}$and $\mathrm{STING}^{+} / \mathrm{CD}_{163^{+}}$cells were significantly increased in livers from NASH patients with advanced fibrosis and correlated only with aggravation of fibrosis stage $(p<0.05)$. Furthermore, compared with controls, NASH patients exhibited significantly increased $\mathrm{STING}{ }^{+} / \mathrm{p}-\mathrm{TBK} 1^{+}$cell numbers. In a coculture system, the amount of p-TBK1 and the mRNAs of IL1 $\beta$ and IL6 in THP1 macrophages, as well as the amount of $\alpha$-SMA and the mRNAs of Col1a1, Fn and TGF $\beta 1$ in LX2 cells were significantly increased upon STING activation in macrophages $(p<0.05)$. Therefore, increased STING expression in MoMFs appears to be indicative of NAFLD progression, and STING could be a new target for NAFLD therapy.
\end{abstract}

These authors contributed equally: Xiaoxiao Wang, Huiying Rao

Supplementary information The online version of this article (https:// doi.org/10.1038/s41374-019-0342-6) contains supplementary material, which is available to authorized users.

Feng Liu

liu1116m@sina.com

$\triangle$ Lai Wei

weilai@pkuph.edu.cn

$1 \quad$ Peking University People's Hospital, Peking University Hepatology Institute, Beijing Key Laboratory of Hepatitis C and Immunotherapy for Liver Diseases, 100044 Beijing, China

2 Department of Pathology, The Fifth Medical Center of PLA General Hospital, 100039 Beijing, China

\section{Introduction}

Nonalcoholic fatty liver disease (NAFLD) is a series of diseases extending from nonalcoholic fatty liver (NAFL, only steatosis) to nonalcoholic steatohepatitis (NASH, steatosis and inflammation) and subsequent fibrosis that

3 Department of Pathology, Yong Loo Lin School of Medicine, National University of Singapore, National University Hospital, 5 Lower Kent Ridge Road, Singapore 119074, Singapore

4 Department of Nutrition and Food Science, Texas A\&M University, College Station, TX 77843, USA

5 Beijing Tsinghua Changgung Hospital, Tsinghua University, 102218 Beijing, China 
lead to cirrhosis and predispose to hepatocellular carcinoma (HCC) [1-4]. It is estimated that $25 \%$ of the general population in the whole world is NAFLD, and the incidence of NAFLD is increasing gradually. The mortality of NAFLD-related liver disease is increased, especially in the stage of advanced fibrosis $[4,5]$. NAFLD is estimated to become the leading cause of liver failure and transplantation worldwide by 2020 [6]. However, there is no approved therapy for NAFLD. Therefore, it is urgent to better understand the pathogenesis of NAFLD/NASH with the hope of developing new therapeutic approaches.

In the liver, accumulation of toxic lipid species initiates or exacerbates the pathogenesis of NAFLD. When lipotoxic species are accumulated excessively, they act as stimulators to provoke endoplasmic reticulum (ER) oxidative stress and promote the development and progression of liver inflammation and fibrosis in NASH [7]. Stimulator of interferon genes (STING), an ubiquitously expressed transmembrane protein on the membrane of ER, can be activated by ER stress [8-10]. Activated STING has been reported to activate the downstream signaling cascades and, consequently, to promote hepatocyte injury and dysfunction in hepatocytes and in a high-fat diet (HFD) mouse model [11-14]. Such evidence suggests that the STING signaling pathway is participated in the progression of NASH. Luo et al. revealed that STING expression in liver sections of NAFLD patients was increased and illustrated that the deleterious role of STING in NASH is mediated by enhancing the proinflammatory reaction of macrophages [15]. Consistently, $\mathrm{Yu}$ et al. validated that STING-mediated inflammation in Kupffer cells (KCs) contributes to NAFLD development in methionine- and choline-deficient diet (MCD) and HFD mouse models [16]. However, the macrophage sources of STING in human liver remain unknown.

Macrophages are a very heterogeneous population in the context of liver diseases [17]. In humans, hepatic macrophages consist of liver resident macrophages, namely $\mathrm{KCs}$, and monocyte-derived macrophages (MoMFs), which are recruited to the liver from peripheral circulation $[18,19]$. When the liver is damaged, activated $\mathrm{KCs}\left(\mathrm{CD} 68^{+}\right.$macrophages) can produce various proinflammatory cytokines and chemokines, leading to inflammatory cells and lymphocytes infiltration and sterile inflammation response [20]. The abundance of CCR2 ${ }^{+}$ MoMFs, S100A9 ${ }^{+}$macrophages (the inflammatory phenotype of $\mathrm{CCR}^{+} \mathrm{MoMFs}$ ) and $\mathrm{CD} 163^{+}$macrophages was increased during the progression of NAFLD, especially in the inflammatory and fibrotic stages of this disease [18, 21, 22]. Hepatic macrophages have been proposed to promote NASH progression through influencing of hepatocyte steatosis, attraction of inflammatory lymphocytes, stimulation of angiogenesis, and/or promotion of liver fibrosis [23-26]. However, the signaling pathway(s) through which macrophages participate in NASH development need to be elucidated. STING is expressed on macrophages and appears to be a critical member of the signaling pathways responsible for NAFLD pathogenesis. To date, however, the cellular sources of $\mathrm{STING}^{+}$macrophages and the relationship of these macrophages with the progression of NAFLD are unknown. In our current research, we examined the cell sources of STING-expressing macrophages and correlated the expression of STING with the progression of NAFLD in 106 human liver samples, and investigated the mechanisms by which STING signaling pathway is involved in NAFLD progression.

\section{Materials and methods}

\section{Human liver samples and preparation}

Formalin-fixed and paraffin-embedded (FFPE) liver tissues were obtained from the Department of Pathology at Peking University People's Hospital and The Fifth Medical Center of PLA General Hospital. This study was approved by the Ethics Committee of both hospitals (2017PHB133-01) and conformed to the ethical guidelines of the 1975 Declaration of Helsinki. All participants have signed the informed consent. Liver tissues included 98 samples from subjects ( $\geq 18$ years of age) with biopsy-proven NAFLD/NASH and 8 samples from healthy donors. Patients with various viral hepatitis, alcoholic liver disease (ALD), drug-induced liver disease, autoimmune liver disease, cholestatic liver disease or hereditary metabolic liver disease were excluded.

Each of the 106 samples was sectioned at $4 \mu \mathrm{m}$ thickness for immunohistochemistry (IHC) and stained with hematoxylin and eosin (H\&E) and Masson trichrome for histological assessment. Liver histology for all cases was evaluated by two specialized pathologists who were blinded to the patient details according to the NASH Clinical Research Network (NASH-CRN) System (steatosis was scored from 0 to 3 , ballooning was scored from 0 to 2 , lobular inflammation was scored from 0 to 3 , portal inflammation was scored from 0 to 2 and liver fibrosis was scored from 0 to 4) [27-29].

\section{Multiplex staining}

To identify STING expression in different liver cells, multiplex immunofluorescence staining was performed on FFPE slides using a PANO 7-plex IHC kit (Panovue, Beijing, China, cat. 0004100100) according to the manufacturer's instructions. The primary antibodies used for staining are listed in Table 1, and the concentration and staining order of the antibodies were optimized in advance. The slides were serially incubated with primary antibodies and horseradish peroxidase-conjugated secondary antibodies and subjected to tyramide signal amplification (TSA). 
Table 1 Summary of primary antibodies used for TSA-IHC

\begin{tabular}{lllll}
\hline $\begin{array}{l}\text { Primary } \\
\text { antibody target }\end{array}$ & Isotype & Supplier & Product no. & Dilution \\
\hline STING & rabbit mAb & CST & $\# 13647$ & $1: 1000$ \\
CD68 & mouse mAb & Origene & ZM-0060 & $\begin{array}{l}\text { Ready-to- } \\
\text { use }\end{array}$ \\
& rabbit mAb & Abcam & ab176390 & $1: 400$ \\
CCR2 & rabbit pAb & Abcam & ab63818 & $1: 400$ \\
S100A9 & rabbit mAb & CST & $\# 93498$ & $1: 800$ \\
CD163 & mouse mAb & Origene & TA500921 & $1: 50$ \\
CD36 & mouse mAb & Origene & TA505384 & $1: 150$ \\
CD1c & mouse mAb & Leica & PA0943 & Ready-to- \\
SMA & & & & use \\
& & & & $1: 100$ \\
phospho-TBK1 & rabbit pAb & Affinity & AF8190 & \\
(Ser 172) & & & &
\end{tabular}

After each round of TSA operation, the slides were heattreated by microwave for antigen retrieval and antibody stripping. After all antigens had been labeled, counterstaining was performed using 4'-6'-diamidino-2-phenylindole (DAPI, Sigma-Aldrich, Missouri, USA, cat. D9542).

\section{Multispectral imaging}

Images were acquired using the Mantra Quantitative Pathology Imaging System (PerkinElmer). Representative regions of interest were chosen, and multiple fields of view were acquired at $\times 200$ magnification as multispectral images.

\section{Image analysis}

The multispectral images were analyzed using InForm image analysis software 2.4 (PerkinElmer). The spectral library was built based on the single-stained slides for each fluorophore, and unstained sections were used to extract the spectrum of autofluorescence of the tissues.

Five fields $(\times 200)$ of each sample were randomly selected and captured for analysis. The number of cells in the portal tract (per portal tract area) and liver lobule tissue were quantified. The numbers of $\mathrm{STING}^{+}$cells and $\mathrm{STING}^{+}$ macrophages in per visual field were shown as the means \pm standard deviations (SDs).

\section{Cells coculture, detection and analysis}

THP1 cells (human monocyte) were differentiated into THP1 macrophages. After differentiation, THP1 macrophages were seeded on six-well upper transwell insert $(0.4$ $\mu \mathrm{M})$ and treated with $2^{\prime} 3^{\prime}$-cyclic guanosine monophosphateadenosine monophosphate (2'3'-cGAMP, $20 \mu \mathrm{g} / \mathrm{mL})$ and/or palmitate (PA, $1 \mathrm{mM}$ ) for $24 \mathrm{~h}$. After stimulation, THP1 macrophages were added to LX2 cells for coculture
Table 2 Summary of primary antibodies used for western blotting

\begin{tabular}{lllll}
\hline $\begin{array}{l}\text { Primary } \\
\text { antibody target }\end{array}$ & Isotype & Supplier & Product no. & Dilution \\
\hline STING & rabbit mAb & CST & 13647 & $1: 1000$ \\
TBK1 & rabbit mAb & CST & 3504 & $1: 1000$ \\
phospho-TBK1 & rabbit mAb & CST & 5483 & $1: 1000$ \\
$\alpha$-SMA & rabbit mAb & CST & 19245 & $1: 1000$ \\
GAPDH & HRP- & Proteintech & HRP-60004 & $1: 5000$ \\
& mouse mAb & & & \\
\hline
\end{tabular}

experiments. After coculturing for 24 or $48 \mathrm{~h}$, supernatants of cocultures were collected. Also, total RNA or protein of THP1 macrophages and/or LX2 cells were extracted and analyzed. Detailed methods were provided in the Supplementary Methods. The information of primary antibodies used for Western Blotting were shown in Table 2.

\section{Statistical analysis}

Statistical analysis was performed using GraphPad Prism Version 5.0a (GraphPad Software Inc., San Diego, CA, USA). Comparisons of various STING $^{+}$macrophage numbers among different NAFLD groups were analyzed by one-way ANOVA. Differences in various $\mathrm{STING}^{+}$macrophage abundance in the liver lobule and portal tract in subgroups were evaluated with the Mann-Whitney $U$ test. Differences in cell protein, mRNA and cytokine levels between two coculture groups were analyzed using the Mann-Whitney $U$ test. Two-sided $p$ values less than 0.05 were considered to indicate significance.

\section{Results}

\section{Summary of the clinical and liver histology data}

All 98 NAFLD patients and 8 healthy control samples were divided into five groups according to the NASH-CRN system, namely the Control, Steatosis, NASH-fibrosis 0 (F0), NASHfibrosis 1-2 (F1-2) and NASH-fibrosis 3-4 (F3-4) groups. Clinical data were summarized in Table 3 . There were 56 females (57\%), with a mean age of 45 years (range: $18-76$ years), among the NAFLD patients. In all, 38 patients (39\%) were overweight, and a further 33 patients (34\%) were obese.

Compared with control samples, liver samples of patients with steatosis showed increased fat deposition, those of NASH patients without fibrosis (NASH-F0) showed increased ballooning degeneration and inflammatory cell infiltration, and those of NASH patients with fibrosis showed increased collagen deposition as indicated by H\&E and Masson trichrome staining (Fig. 1), confirming the existence of liver steatosis, inflammation and/or fibrosis. 
Table 3 Clinical and histologic data for human NAFLD patients and healthy controls

\begin{tabular}{|c|c|c|c|c|c|}
\hline & $\begin{array}{l}\text { Control } \\
(n=8)\end{array}$ & $\begin{array}{l}\text { Steatosis } \\
(n=10)\end{array}$ & $\begin{array}{l}\text { NASH-F0 } \\
(n=17)\end{array}$ & $\begin{array}{l}\text { NASH-F1-2 } \\
(n=51)\end{array}$ & $\begin{array}{l}\text { NASH-F3-4 } \\
(n=20)\end{array}$ \\
\hline $\operatorname{Sex}(F / M)$ & $5 / 3$ & $3 / 7$ & $6 / 11$ & $29 / 22$ & $18 / 2$ \\
\hline Age $^{\mathrm{a}}$, year & $41 \pm 21$ & $40 \pm 15$ & $34 \pm 13$ & $49 \pm 21$ & $61.5 \pm 10$ \\
\hline $\mathrm{BMI}^{\mathrm{a}, \mathrm{b}}, \mathrm{kg} / \mathrm{m}^{2}$ & $23.88 \pm 4.22$ & $26.46 \pm 2.05$ & $24.09 \pm 3.04$ & $27.05 \pm 4.45$ & $26.71 \pm 4.71$ \\
\hline $\mathrm{ALT}^{\mathrm{a}}, \mathrm{U} / \mathrm{L}$ & $25 \pm 11$ & $34 \pm 42$ & $78 \pm 99$ & $64 \pm 76$ & $46 \pm 57$ \\
\hline $\mathrm{AST}^{\mathrm{a}}, \mathrm{U} / \mathrm{L}$ & $26 \pm 12$ & $23 \pm 13$ & $41 \pm 35$ & $49 \pm 43$ & $52 \pm 39$ \\
\hline $\mathrm{GGT}^{\mathrm{a}}, \mathrm{U} / \mathrm{L}$ & $42 \pm 26$ & $36 \pm 58$ & $49 \pm 63$ & $59 \pm 65$ & $75 \pm 77$ \\
\hline $\mathrm{Glu}^{\mathrm{a}}, \mathrm{mg} / \mathrm{dL}$ & $5.10 \pm 0.68$ & $4.52 \pm 0.42$ & $4.9 \pm 0.69$ & $5.17 \pm 1.70$ & $5.10 \pm 1.28$ \\
\hline $\mathrm{TG}^{\mathrm{a}}, \mathrm{mg} / \mathrm{dL}$ & $1.45 \pm 1.51$ & $1.33 \pm 0.49$ & $3.97 \pm 2.41$ & $1.96 \pm 2.38$ & $1.58 \pm 1.53$ \\
\hline \multicolumn{6}{|l|}{ Liver histology $\mathrm{y}^{\mathrm{a}}$} \\
\hline Steatosis & $0 \pm 0$ & $1 \pm 0$ & $1 \pm 0$ & $1 \pm 1$ & $1 \pm 0$ \\
\hline Lobular inflammation & $0 \pm 0$ & $0 \pm 0$ & $1 \pm 0$ & $1 \pm 1$ & $1 \pm 1$ \\
\hline Portal inflammation & $0 \pm 0$ & $0 \pm 0$ & $0 \pm 0$ & $1 \pm 1$ & $2 \pm 0$ \\
\hline Fibrosis & $0 \pm 0$ & $0 \pm 0$ & $0 \pm 0$ & $1 \pm 1$ & $4 \pm 1$ \\
\hline
\end{tabular}

$F$ female, $M$ male, $B M I$ body mass index, $A L T$ alanine transaminase, $A S T$ aspartate transaminase, $G G T$ gamma-glutamyl transferase, $T G$ triglyceride, $I Q R$ interquartile range, $F$ fibrosis

${ }^{\mathrm{a}}$ Clinical data and liver histology scores are presented as the median $\pm \mathrm{IQR}$

${ }^{\mathrm{b}}$ BMI-CHINA Chinese criteria [48]

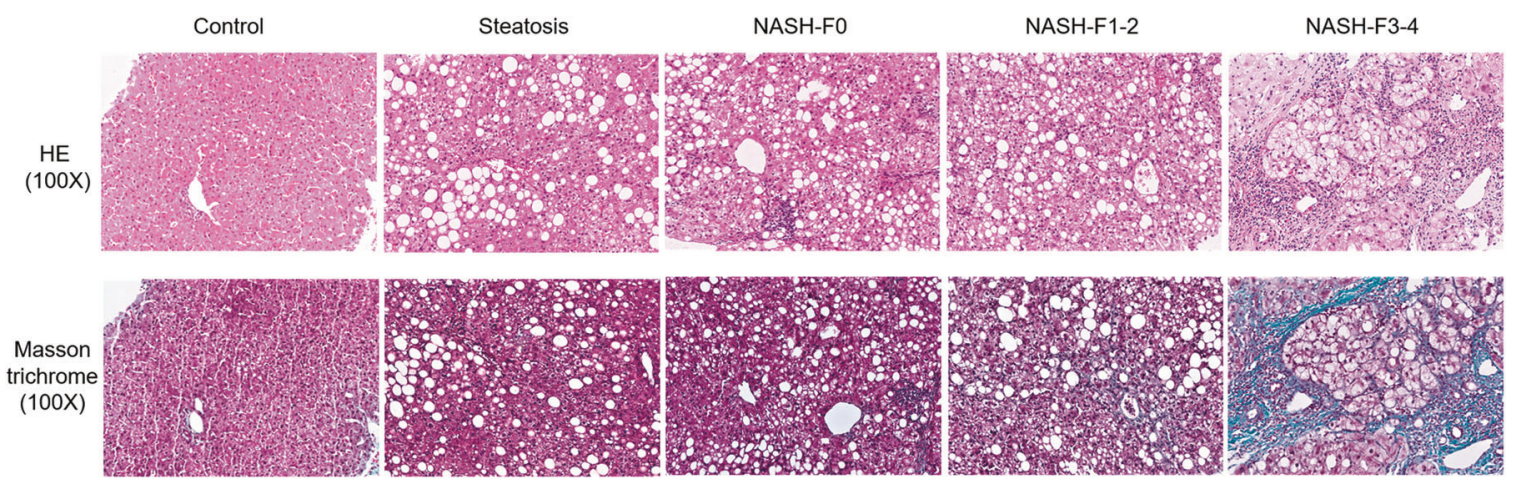

Fig. 1 NAFLD patients showed increased fat deposition, inflammatory cell infiltration, ballooning degeneration and collagen production. Liver sections from controls and patients with NAFLD were stained

\section{NASH patients showed significantly increased liver STING $^{+}$cells, which were increased with the aggravation of inflammation and liver fibrosis}

To address the relevance between STING and human NAFLD progression, we analyzed STING expression in liver biopsies of controls and NAFLD patients ranging from steatosis to NASH-F0, NASH-F1-2 and NASH-F3-4 groups (Table 3 ). STING $^{+}$cells were scattered in the liver lobule and portal tract, and the intensity of staining in livers of NASH patients was much stronger than that in livers of controls (Fig. 2a). Compared with healthy controls, NASH patients exhibited increased $\mathrm{STING}^{+}$cell populations (all $p<0.05$ ), especially NASH patients with fibrosis (NASH-F1-2 vs. control, $p=0.019$; NASH-F3-4 vs. with $H \& E$ (top row, magnification $\times 100$ ) and Masson trichrome (bottom row, magnification $\times 100$ )

control, $p<0.001$ ) (Fig. 2b), and the increased STING ${ }^{+}$cell numbers were mainly located in the portal tract (all $p<$ 0.05) (Fig. 2c). Compared with NASH patients with fibrosis stage of 1-2, NASH patients with fibrosis stage of 3-4 exhibited a greater increase in the numbers of $\mathrm{STING}^{+}$cells in the portal tract $(p<0.001)$ (Fig. 2c).

To verify the relevance of the expression of STING to the progression of steatosis, inflammation and fibrosis, we analyzed the numbers of STING ${ }^{+}$cells in liver samples at different steatosis, lobular inflammation, portal inflammation grades and fibrosis stages among patients compared with the other liver histology scores (Supplementary Table 2: Table S2). In Lobular inflammation (LI) group 1, compared with NAFLD patients whose lobular inflammation score was 0 , patients whose lobular inflammation score 


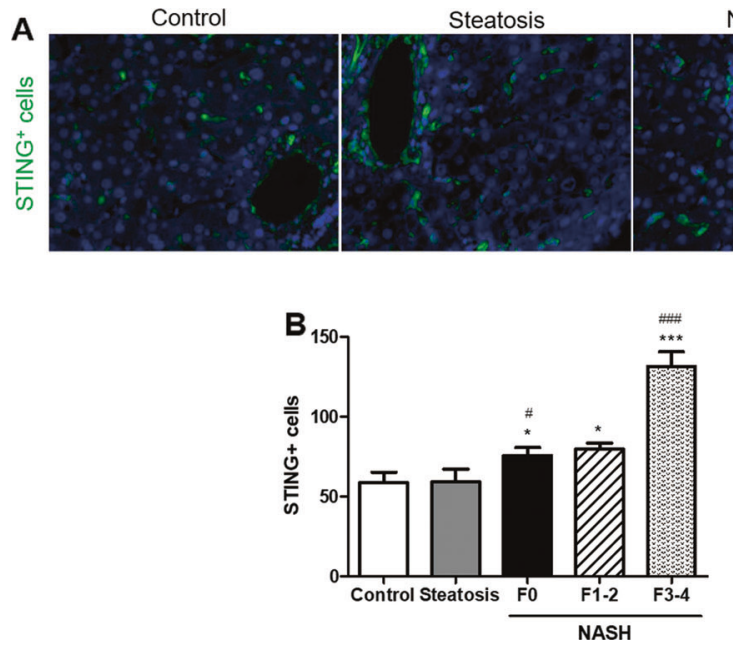

Fig. 2 Liver sections from NASH patients showed increased STING ${ }^{+}$ cells. a Representative images of liver sections from patients with NAFLD $(n=98)$ and controls $(n=8)$ that were stained for STING (green). Quantification of STING ${ }^{+}$cells by TSA-IHC staining in the whole section field (b), and in the portal tract and liver lobule (c). For
NASH-FO
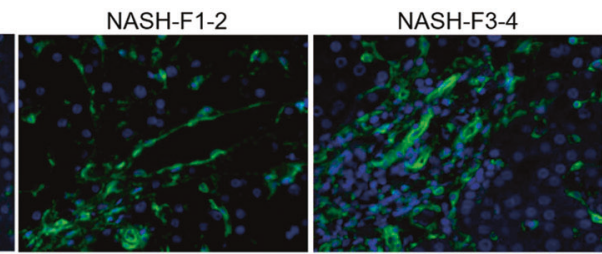

Liver Lobule

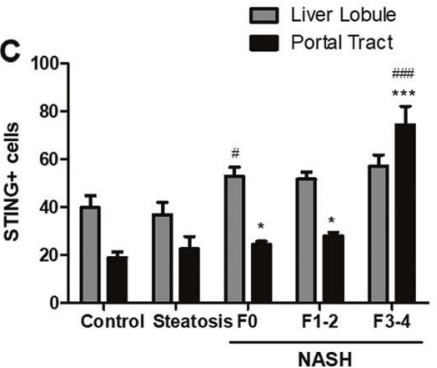

each sample, the $\mathrm{STING}^{+}$cells (green) were counted in five highpower fields; magnification $\times 200$. $* p<0.05$, $* * * p \leq 0.001$ : patients with different stages of NAFLD vs. controls. ${ }^{\#} p<0.05,{ }^{\# \# \#} p \leq 0.001$ : comparison between two closer NAFLD stages was 1 exhibited increased STING $^{+}$cell numbers in the liver lobule ( $p=0.008$ ) (Supplementary Fig. 1: Fig. S1A). In LI group 2 and Fibrosis (F) group 1, compared with patients whose lobular inflammation grade was 1 and fibrosis stage was 1-2, patients whose lobular inflammation grade was 2-3 and fibrosis stage was 3-4 had increased $\mathrm{STING}^{+}$cell numbers in both the liver lobule and portal tract (all $p<$ 0.05) (Supplementary Fig. 1: Fig. S1B-1C). In F group 2, compared with patients whose fibrosis stage was 0 or $1-2$, patients whose fibrosis stage was 3-4 exhibited increased $\mathrm{STING}^{+}$cell populations, mainly in the portal tract $(p=$ 0.017, $p<0.001$ ) (Supplementary Fig. 1: Fig. S1D).

By TSA-IHC, we observed that STING was mainly expressed in macrophages $\left(\mathrm{CCR} 2^{+}, \mathrm{S} 100 \mathrm{~A} 9^{+}, \mathrm{CD} 68^{+}\right.$and $\mathrm{CD}_{163}{ }^{+}$cells), although it was poorly expressed in liver sinusoidal endothelial cells (LSECs, CD $36^{+}$cells), dendritic cells (DCs, $\mathrm{CD}^{+}{ }^{+}$cells) and hepatic stellate cells (HSCs, $\mathrm{SMA}^{+}$cells) (Supplementary Fig. 2: Fig. S2A-2B). To further characterize the dynamics of STING in NAFLD, STING expression in different macrophages during NAFLD progression needs to be clarified.

\section{NASH patients with fibrosis showed increased liver STING $^{+} /$CCR2 $^{+}$and STING ${ }^{+} /$S100A9 $^{+}$cells, which were elevated with the aggravation of inflammation and fibrosis}

To investigate the relevance of STING in $\mathrm{CCR} 2^{+}$cells to human NAFLD progression, we analyzed $\mathrm{STING}^{+} / \mathrm{CCR} 2^{+}$ cells in healthy controls and NAFLD patients. Compared with healthy controls, NAFLD patients exhibited increased
$\mathrm{STING}^{+} / \mathrm{CCR} 2^{+}$cell numbers in the liver (Fig. 3a), especially NASH patients with fibrosis (NASH-F1-2 vs. control, $p=0.006$; NASH-F3-4 vs. control, $p<0.001$ ) (Fig. 3c). In NASH patients with fibrosis stage of 1-2, increased numbers of STING ${ }^{+} / \mathrm{CCR}^{+}{ }^{+}$cells were mainly observed in the liver lobule $(p=0.006)$; in NASH patients with fibrosis stage of 3-4, the increased numbers existed in both the liver lobule $(p=0.033)$ and the portal tract $(p<0.001)$ (Fig. 3d). Strikingly, compared with NASH patients with fibrosis stage of 1-2, NASH patients with fibrosis stage of 3-4 had a greater increase in the numbers of $\mathrm{STING}^{+} / \mathrm{CCR} 2^{+}$cells in the portal tract $(p<0.001)$ (Fig. 3d).

$\mathrm{S} 100 \mathrm{~A} 9^{+}$cells are a proinflammatory cellular phenotype of $\mathrm{CCR}^{+}$cells. To further characterize the infiltrating $\mathrm{CCR} 2^{+}$cells, we analyzed the expression of STING on S100A $9^{+}$cells. Compared with controls, NAFLD patients exhibited increased $\mathrm{STING}^{+} / \mathrm{S} 100 \mathrm{~A} 9^{+}$cell populations in the liver (Fig. 3b), especially NASH patients with fibrosis stage of 3-4 (NASH-F3-4 vs. control, $p=0.009$ ) (Fig. 3e). The numbers of $\mathrm{STING}^{+} / \mathrm{S} 100 \mathrm{~A} 9^{+}$cells were increased mainly in the portal tract (NASH-F1-2 vs. control, $p=$ 0.004; NASH-F3-4 vs. control, $p=0.002$ ) (Fig. 3f). Remarkably, compared with NASH patients with mild fibrosis, NASH patients with advanced fibrosis exhibited a greater increase in the numbers of $\mathrm{STING}^{+} / \mathrm{S} 100 \mathrm{~A} 9^{+}$cells in the portal tract $(p<0.001)$ (Fig. 3f).

The expression of STING in CCR2 ${ }^{+}$cells and S100A9 $9^{+}$ cells was analyzed at different inflammation grades and fibrosis stages (Supplementary Table 2: Table S2).

In LI group 2, compared with patients whose lobular inflammation grade was 1, patients whose lobular inflammation 


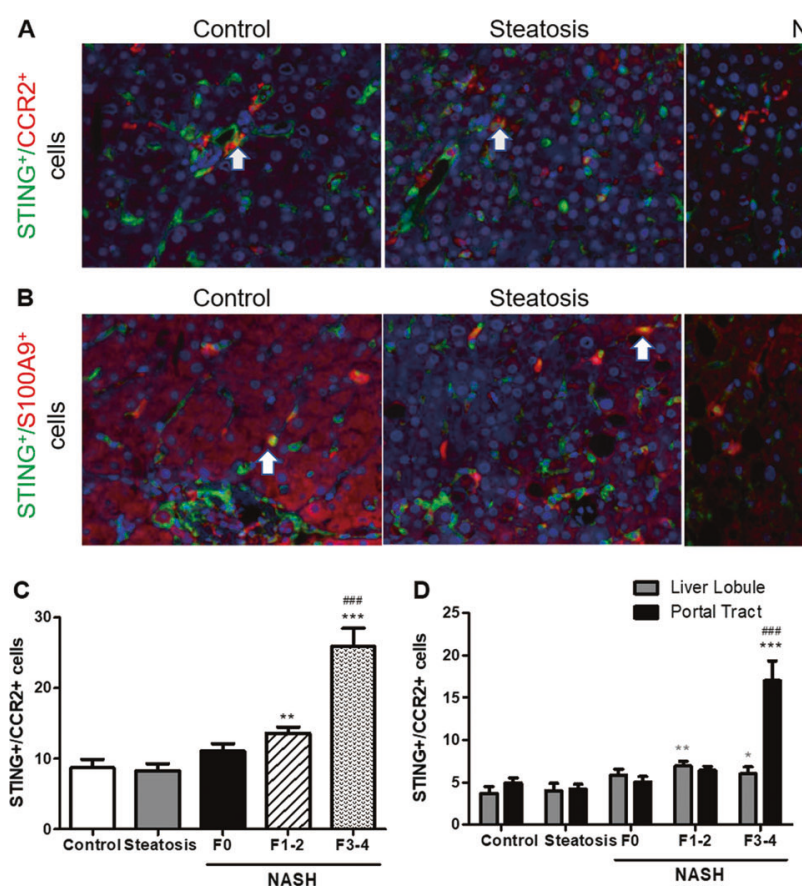

Fig. 3 Liver sections from NASH patients with fibrosis showed increased $\mathrm{STING}^{+} / \mathrm{CCR}^{+}$cells and $\mathrm{STING}^{+} / \mathrm{S} 100 \mathrm{~A} 9^{+}$cells. $\mathbf{a}, \mathbf{b}$ Representative images of liver sections from patients with NAFLD $(n=98)$ and controls $(n=8)$ that were stained for STING (green), CCR2 (red) and S100A9 (red). Quantification of STING ${ }^{+} / \mathrm{CCR}^{+}$cells and $\mathrm{STING}^{+} / \mathrm{S} 100 \mathrm{~A} 9^{+}$cells (yellow, white arrows) by TSA-IHC

grade was 2-3 displayed increased numbers of $\mathrm{STING}^{+} /$ $\mathrm{CCR}^{+}$cells and $\mathrm{STING}^{+} / \mathrm{S} 100 \mathrm{~A} 9^{+}$cells in both the liver lobule and the portal tract (all $p<0.05$ ) (Supplementary Figs. 3-4: Figs. S3A, S4A). In Portal inflammation (PI) group 1, compared with patients whose portal inflammation grade was 0 , patients whose portal inflammation grade was 1-2 exhibited increased numbers of $\mathrm{STING}^{+} / \mathrm{CCR} 2^{+}$cells in both the liver lobule and the portal tract, and increases in the $\mathrm{STING}^{+} / \mathrm{S} 100 \mathrm{~A} 9^{+}$cell populations were observed mainly in the portal tract (all $p<0.05$ ) (Supplementary Figs. 3-4: Figs. S3B, S4B). In F groups 1 and 2, compared with the patients whose fibrosis stage was 1-2 or 0 , patients whose fibrosis stage was 3-4 displayed increased numbers of $\mathrm{STING}^{+} / \mathrm{CCR} 2^{+}$and $\mathrm{STING}^{+} / \mathrm{S} 100 \mathrm{~A} 9^{+}$cells, mainly in the portal tract (all $p<0.05$ ) (Supplementary Figs. 3-4: Figs. S3C-3D, S4C-4D).

\section{NASH patients with advanced fibrosis showed increased liver STING ${ }^{+} / \mathrm{CD} 68^{+}$and $\mathrm{STING}^{+} / \mathrm{CD} 163^{+}$ cells, which were elevated with the aggravation of fibrosis}

KCs were indicated by the marker CD68. To investigate the relevance of STING in $\mathrm{CD}^{+} 8^{+}$cells to human NAFLD progression, we analyzed $\mathrm{STING}^{+} / \mathrm{CD} 8^{+}$cells in livers of healthy controls and NAFLD patients. $\mathrm{STING}^{+} / \mathrm{CD}^{+} 8^{+}$
NASH-FO

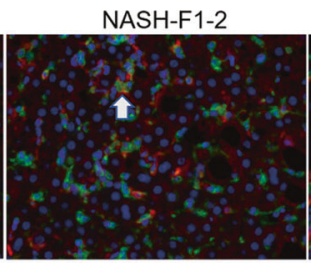

NASH-F1-2

NASH-FO
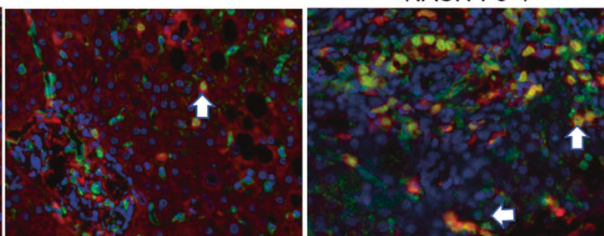

NASH-F3-4
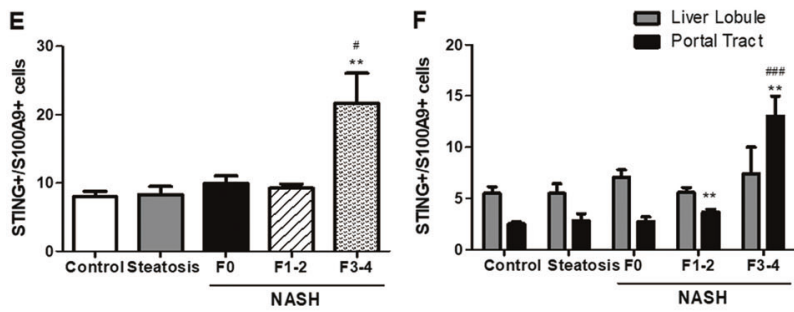

staining in the whole liver field (c, e), and in the portal tract and liver lobule $(\mathbf{d}, \mathbf{f})$. For each sample, the $\mathrm{STING}^{+} / \mathrm{CCR}^{+}$cells and $\mathrm{STING}^{+}$/ S100A9 ${ }^{+}$cells (yellow, white arrows) were counted in five high-power fields; magnification $\times 200$. $* p<0.05$, ** $p \leq 0.01$ and $* * * p \leq 0.001$ : patients with different stages of NAFLD vs. controls. ${ }^{\#} p<0.05,{ }^{\# \# \#} p \leq$ $0.001^{*}$ comparison between two closer NAFLD stages. $\mathrm{F}$ fibrosis

cells were scattered in livers of healthy controls and NAFLD patients and were mainly located in the liver lobule (Fig. 4a). Compared with healthy controls, only NASH patients with fibrosis stage of 3-4 exhibited increased numbers of STING ${ }^{+} / \mathrm{CD}^{+} 8^{+}$cells (NASH-F3-4 vs. control, $p=0.030$ ) (Fig. 4d), and the increased $\mathrm{STING}^{+} / \mathrm{CD} 8^{+}$cell populations were largely present in the portal tract (NASHF3-4 vs. control, $p<0.001$ ) (Fig. 4e). Remarkably, compared with NASH patients with fibrosis stage of 1-2, NASH patients with advanced fibrosis displayed a greater increase in the numbers of $\mathrm{STING}^{+} / \mathrm{CD}^{+} 8^{+}$cells in the portal tract $(p<0.001)$ (Fig. 4e).

CD163 is a marker of anti-inflammatory macrophages, and liver biopsy stained by TSA-IHC revealed that nearly all $\mathrm{CD}^{+} 8^{+}$cells expressed CD163 (Fig. 4b), thereby indicating that STING expression in $\mathrm{CD}_{163}{ }^{+}$cells may be similar to that in $\mathrm{CD}^{+} 8^{+}$cells. We selected 24 samples from all participants to explore STING expression in $\mathrm{CD} 163^{+}$cells during NASH development. STING ${ }^{+} / \mathrm{CD} 163^{+}$cells were mainly located in the liver lobule (Fig. 4c). Compared with healthy controls, only NASH patients with advanced fibrosis displayed increased numbers of STING ${ }^{+} / \mathrm{CD} 63^{+}$cells (NASHF3-4 vs. control, $p=0.009$ ) (Fig. 4f). The increased numbers of $\mathrm{STING}^{+} / \mathrm{CD}_{163}{ }^{+}$cells were mainly in the portal tract (NASH-F1-2 vs. control, $p<0.001$; NASH-F3-4 vs. control, $p=0.033$ ) (Fig. 4g). Remarkably, compared with NASH 

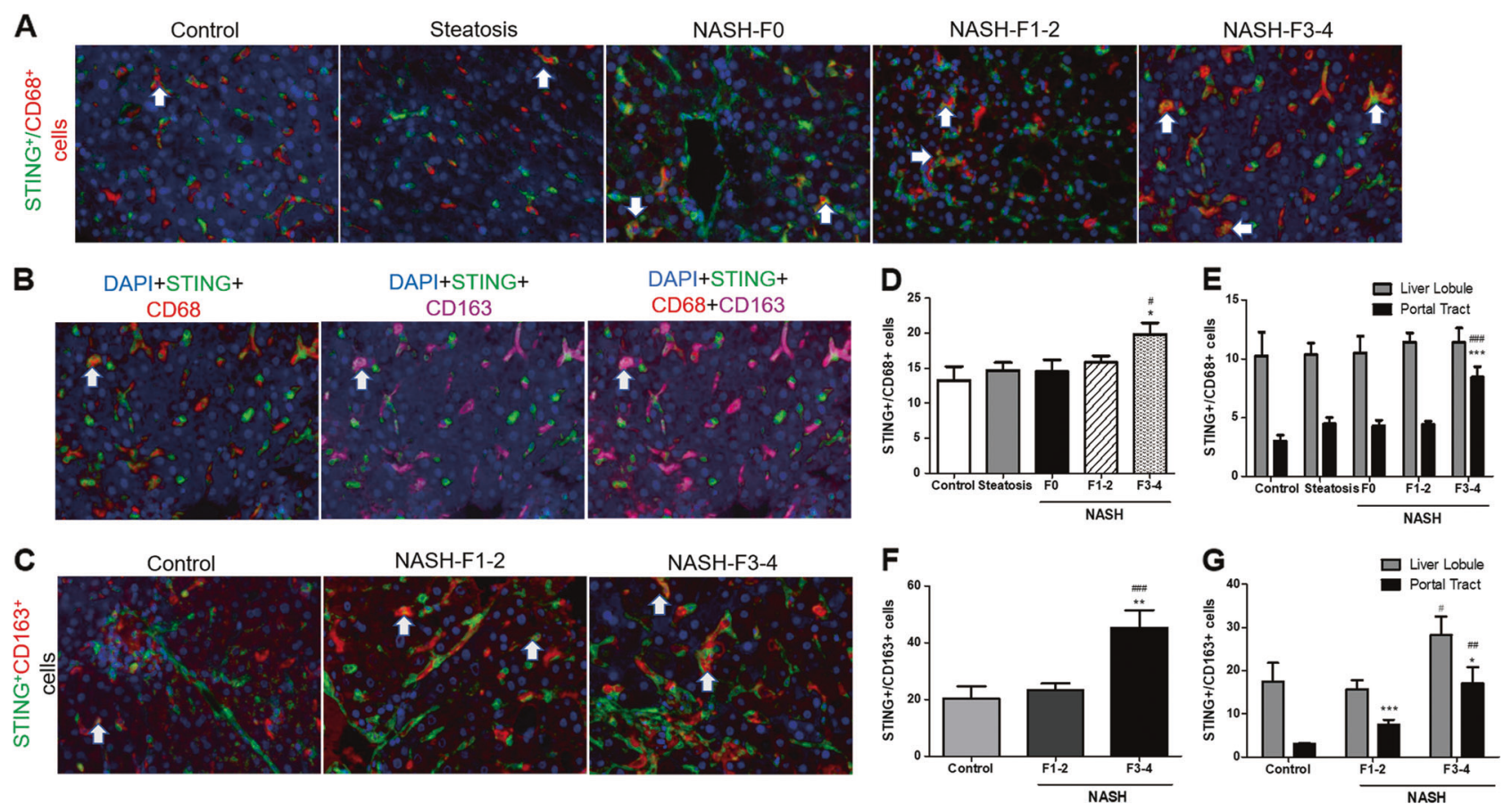

Fig. 4 Liver sections from NASH patients with fibrosis showed increased $\mathrm{STING}^{+} / \mathrm{CD}^{+} 8^{+}$cells and $\mathrm{STING}^{+} / \mathrm{CD} 163^{+}$cells. a Representative images of liver sections from patients with NAFLD $(n=98)$ and controls $(n=8)$ that were stained for STING (green) and CD68 (red). b Representative images of liver sections from patients with NAFLD that were stained for STING (green), CD68 (red) and CD163 (magenta). c Representative images of liver sections from patients with NAFLD $(n=19)$ and controls $(n=5)$ that were stained for STING (green) and CD163 (red). Quantification of $\mathrm{STING}^{+}$/

patients with fibrosis stage of 1-2, NASH patients with fibrosis stage of 3-4 exhibited a greater increase in the numbers of $\mathrm{STING}^{+} / \mathrm{CD} 163^{+}$cells in both the liver lobule $(p=$ 0.013) and the portal tract $(p=0.004)$ (Fig. 4g).

In $\mathrm{F}$ groups 1 and 2, compared with patients whose fibrosis stage was 1-2 or 0, patients whose fibrosis stage was 3-4 displayed a significant increase in the numbers of $\mathrm{STING}^{+} / \mathrm{CD}^{+} 8^{+}$cells, mainly in the portal tract (both $p<$ 0.05) (Supplementary Fig. 5: Fig. S5A-5B). In F group 2, compared with patients whose fibrosis stage was 1-2, patients whose fibrosis stage was 3-4 also displayed a significant increase in the numbers of $\mathrm{STING}^{+} / \mathrm{CD} 163^{+}$cells, mainly in the liver lobule $(p=0.031)$ (Supplementary Fig. 5: Fig. S5C). There were no differences in the numbers of $\mathrm{STING}^{+} / \mathrm{CD}^{+} 8^{+}$cells or $\mathrm{STING}^{+} / \mathrm{CD} 163^{+}$cells in either the LI or PI groups.

\section{STING induced macrophage activation through TBK1 phosphorylation in NASH patients}

To investigate the mechanisms underlying the role of the STING signaling pathway in NASH progression, we stained liver sections for phospho-TANK-binding kinase 1
$\mathrm{CD} 8^{+}$cells and $\mathrm{STING}^{+} / \mathrm{CD} 163^{+}$cells (yellow, white arrows) by TSA-IHC staining in the whole section field $(\mathbf{d}, \mathbf{f})$, and in the portal tract and liver lobule $(\mathbf{e}, \mathbf{g})$. For each sample, $\mathrm{STING}^{+} / \mathrm{CD}^{+} 8^{+}$cells and $\mathrm{STING}^{+} / \mathrm{CD}_{163}{ }^{+}$cells (yellow, white arrows) were counted in five high-power fields; magnification $\times 200$. $* p<0.05$, $* * p \leq 0.01$ and $* * * p \leq 0.001$ : patients with different stages of NAFLD vs. controls. ${ }^{\#} p<0.05,{ }^{\# \#} p \leq 0.01$ and ${ }^{\# \# \# p} p 0.001$ : comparison between two closer NAFLD stages

(p-TBK1), a downstream protein activated by STING. In healthy controls and NASH patients, TBK1 was phosphorylated in nonparenchymal cells, especially in macrophages. Furthermore, STING was found colocalized with activated TBK1 (p-TBK1). Compared with healthy controls, NASH patients with mild or advanced fibrosis exhibited increased numbers of $\mathrm{STING}^{+} / \mathrm{p}-\mathrm{TBK} 1^{+}$cells (Fig. 5a). Compared with patients whose lobular inflammation grade is 1 (NASH-LI1), numbers of $\mathrm{STING}^{+} / \mathrm{p}$ $\mathrm{TBK}^{+}{ }^{+}$cells were increased in NASH patients whose lobular inflammation grade is 2 (NASH-LI2) (Fig. 5b). These results suggest that the STING-TBK1 signaling pathway in macrophages was activated during $\mathrm{NASH}$.

\section{TBK1 phosphorylation induced upon STING activation in macrophages promoted the production of proinflammatory cytokines and activation of HSCs}

To illustrate the extent to which the STING signaling pathway in macrophages is involved in NASH progression, we examined the effect of STING-driven macrophage activation on HSCs responses in vitro. In cocultures, 
Fig. 5 TBK1 was significantly activated and colocalized with STING in liver sections of NASH patients.

a Representative images of stained liver sections from healthy controls and NASH patients with fibrosis.

b Representative images of stained liver sections from NASH patients whose inflammation grade was 1 and 2 . $\mathrm{STING}^{+}$cells (green, white arrows) and $\mathrm{STING}^{+} / \mathrm{p}-\mathrm{TBK} 1^{+}$ cells (red + green, white arrows) by TSA-IHC staining were shown in the whole liver field; magnification $\times 200$

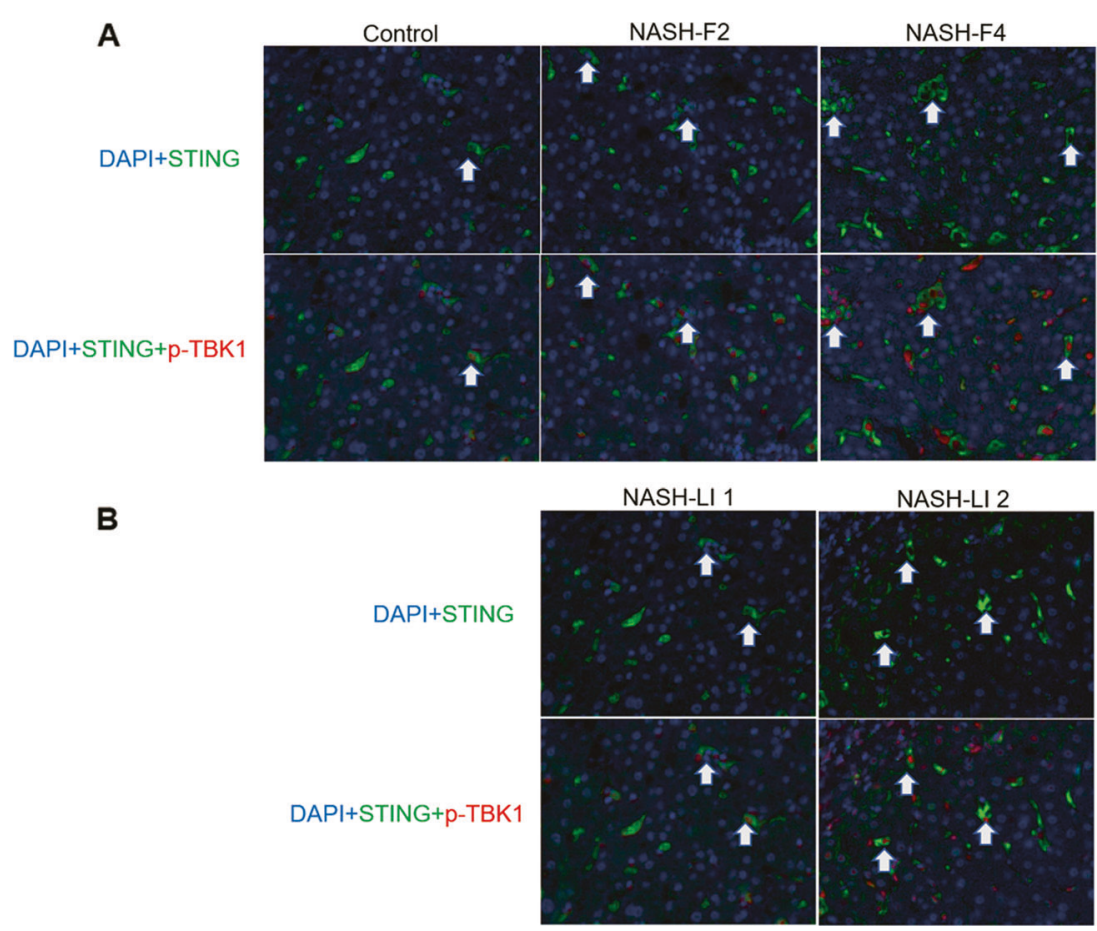

expression of STING in MoMFs was positively correlated with liver inflammation grade and fibrosis stage in human NAFLD. In addition, activation of STING signaling pathway in macrophages enhanced inflammatory responses and increased HSC fibrogenic reaction in vitro. Therefore, we demonstrated that STING appears to promote NAFLD progression through MoMFs.

STING is an essential regulator of the innate immune signaling pathway [8]. The STING signaling pathway is also involved in endothelial inflammation in diet-induced obesity, hepatocyte apoptosis in ALD and hepatocellular death in liver fibrosis in mouse models [10, 11, 30]. In the liver, STING is mainly expressed in nonparenchymal cells, especially macrophages $[9,13,14]$. In our study, we observed that STING expression in liver tissues was associated with liver inflammation and fibrosis progression in NAFLD patients. Of note, STING was mainly expressed in MoMFs $\left(\mathrm{CCR} 2^{+}\right.$and ${\mathrm{S} 100 \mathrm{~A} 9^{+}}^{+}$macrophages), $\mathrm{CD}^{+}$ macrophages and $\mathrm{CD}_{163^{+}}$macrophages, as indicated by the results from TSA-IHC. Luo et al. found that STING was associated with hepatic inflammation in very few human NAFLD samples and showed that STING was mainly expressed in immune cells, including macrophages. However, their study did not address the sources of STINGexpressing cells [15]. Owing to the role of macrophages in regulating the pathogenesis and progression of NAFLD by stimulating hepatocyte steatosis and inflammation and promoting liver fibrosis [23-26, 31], we postulated that $\mathrm{STING}^{+}$macrophages were involved in liver inflammation
Our research suggested that STING was associated with NAFLD development. Notably, STING was mainly expressed in macrophages, especially in MoMFs, and the 


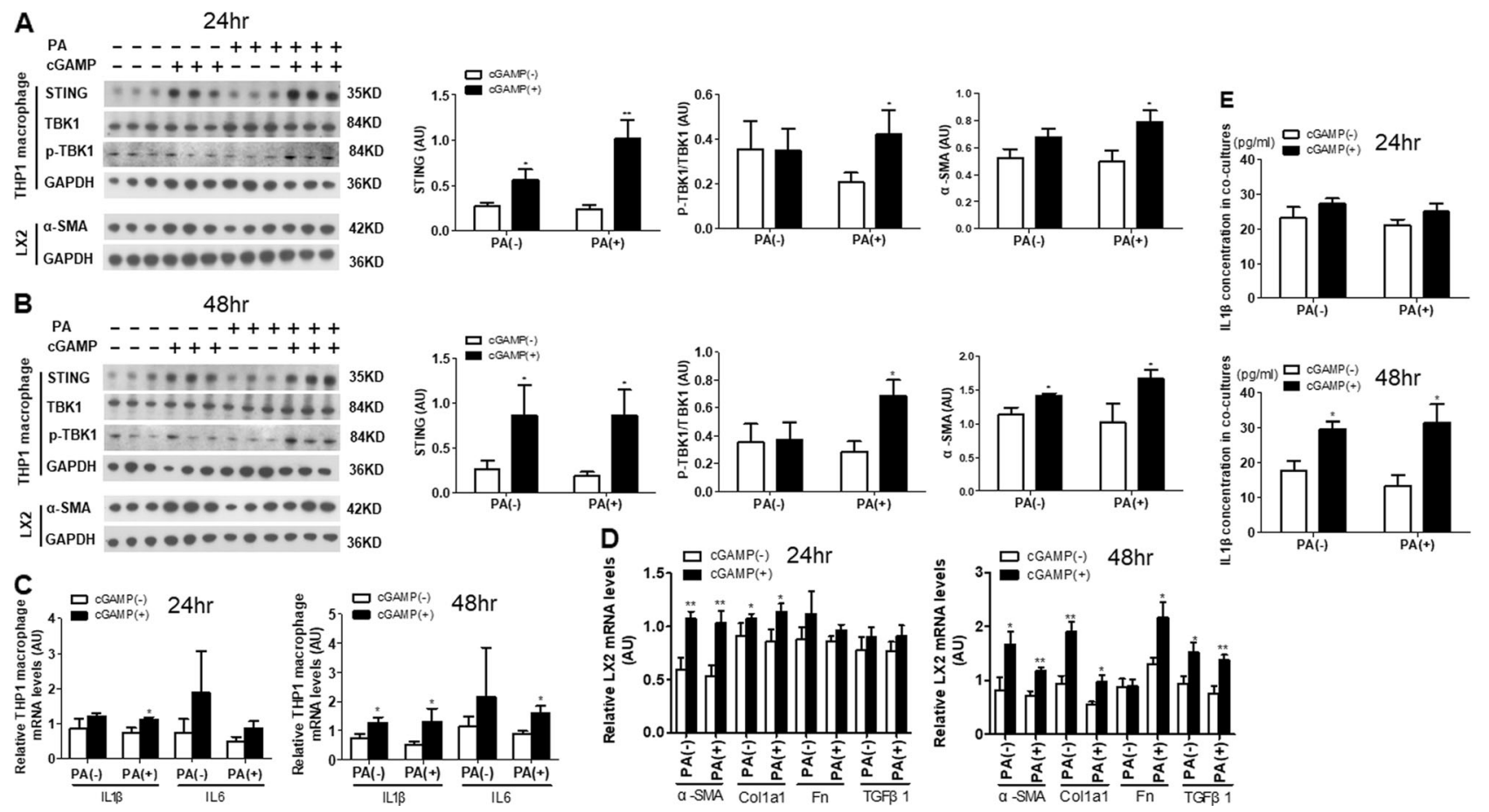

Fig. 6 Phosphorylation of TBK1 in macrophages enhanced proinflammatory cytokine production and HSC activation in vitro. $\mathbf{a}, \mathbf{b}$ The amount of STING and p-TBK1 in THP1 macrophages (a) and $\alpha$-SMA in LX2 cells (b) of cocultures. $\mathbf{c}$ The relative mRNA levels of IL1 $\beta$ and IL6 in cGAMP/PA-treated THP1 macrophages of cocultures. d The relative mRNA levels of $\alpha$-SMA, Col1a1, Fn and TGF $\beta 1$ in LX2 cells

and fibrosis aggravation in human NAFLD development. This was indeed the case according to the findings of this study (see below).

During acute or chronic liver injury, MoMFs represent the predominant macrophage populations that are attracted through CCR2 to the liver injury sites [26, 32, 33]. In the human livers, CCR2 is expressed in all MoMFs [34]. In our study, we showed that the numbers of $\mathrm{STING}^{+} / \mathrm{CCR} 2^{+}$and $\mathrm{STING}^{+} / \mathrm{S} 100 \mathrm{~A}^{+}$macrophages were increased in NASH patients with fibrosis and that the numbers of these two types of macrophages were increased in livers with aggravation of liver inflammation and fibrosis. Regarding to the relationship between MoMFs and liver inflammation, S100A9 is expressed at the early stage of monocytes differentiating to macrophages, and $\mathrm{S} 100 \mathrm{~A}^{+}$cells are the inflammatory cellular phenotype of $\mathrm{CCR}^{+}$macrophages [33, 35-37]. For instance, CCR2 ${ }^{+}$and S100A $9^{+}$proinflammatory MoMFs are abundantly present in the liver portal tract in acetaminophen-induced acute liver failure (AALF) patients [36]. With regard to the relationship between MoMFs and liver fibrosis, CCR2 ${ }^{+}$and S100A9 ${ }^{+}$ cells are significantly increased in NASH patients with fibrosis, and inhibition of infiltrating macrophages with cenicriviroc (a CCR2/CCR5 antagonist) is associated with reduced fibrosis $[21,38]$. In our study, we observed that the increases in $\mathrm{STING}^{+} / \mathrm{CCR}^{+}$and $\mathrm{STING}^{+} / \mathrm{S} 100 \mathrm{~A} 9^{+}$macrophage of cocultures. e The concentrations of IL $1 \beta$ in conditioned media of cocultures of cGAMP/PA-treated THP1 macrophages and LX2 cells. For $(\mathbf{a}-\mathbf{e})$, LX2 cells were cocultured with cGAMP- and/or PA-treated THP1 macrophages for 24 or $48 \mathrm{~h} . * p<0.05$, **p ${ }^{*} \leq 0.01$. AU arbitrary unit

numbers were associated with inflammation and fibrosis aggravation in NASH patients. Thus, it was very likely that STING-expressing MoMFs played an important role in NAFLD liver inflammation and fibrosis progression.

Liver resident macrophages are $\mathrm{CD} 68^{+}$cells, which are critical sentinels that ensure liver homeostasis [35]. In the present study, we observed that $\mathrm{STING}^{+} / \mathrm{CD}^{+} 8^{+}$and $\mathrm{STING}^{+} / \mathrm{CD} 163^{+}$macrophage populations were increased in NASH patients with fibrosis and that the numbers of these two types of macrophages were increased with aggravation of liver fibrosis. This finding implies a likely contribution of STING-expressing $\mathrm{CD}^{+} 8^{+}$cells to NASH progression and is consistent with the findings of a study by Gadd et al. The latter revealed that $\mathrm{CD}^{+} 8^{+}$cell counts increased according to the progression of NASH, especially in fibrosis stage of 2-4 [39]. In the liver, KCs express CD163, a marker of M2 macrophages, which can increase fibrogenesis and tissue remodeling [36, 40-42]. Moreover, $\mathrm{CD}_{163}{ }^{+}$cells cluster in livers of NASH patients and increase in NASH patients with increasing fibrotic or cirrhotic stage [21, 22]. In our study, the increases in $\mathrm{STING}^{+}$/ $\mathrm{CD}^{+} 8^{+}$and $\mathrm{STING}^{+} / \mathrm{CD} 163^{+}$macrophage numbers were correlated with increases in fibrosis stage. Thus, it is likely that STING-expressing KCs also participated in NAFLD fibrosis progression. 
MoMFs can release proinflammatory and profibrogenic cytokines, which in turn promote inflammatory reactions, activate HSCs and increase the production of collagen in chronically inflamed livers [18, 43]. Consistent with this mechanism, activation of the STING signaling pathway in MoMFs appeared to induce cytokine production, which in turn promoted liver inflammation and fibrosis. In our study, the amount of p-TBK1, a downstream activated marker of STING, was significantly increased in liver sections of NASH patients compared to sections from controls. Additionally, p-TBK1 was colocalized with STING in nonparenchymal cells, suggesting STING induction of TBK1 activation. To validate a role for STING-TBK1 signaling in NAFLD, we also performed macrophage-HSC cocultures, and showed that the mRNA levels of proinflammatory factors in THP1 macrophages and the protein and mRNA levels profibrogenic mediators in HSCs were increased upon STING activation in THP1 macrophages. It has been well documented that STING is an essential adaptor protein in the DNA sensing pathway. In response to stimulation by dinucleotides, STING residing in the ER translocates into the Golgi apparatus, where it interacts with TBK1 and induces TBK1 activation [44-47]. Considering this, it is conceivable that activation of the STING-TBK1 signaling pathway was involved in NASH development.

The present study was not able to address the sources of the stimuli or specific signaling pathways that led to STING activation in the liver biopsy samples. In addition, future studies are needed to address how the factors released by macrophages upon STING activation promote NAFLD progression.

In conclusion, we demonstrated that the expression of STING in MoMFs was correlated with liver inflammation and fibrosis progression while confirming the relevance of STING to human NAFLD. Therefore, STING expression may predict the status of NASH. Additionally, targeting STING to inhibit MoMFs activation is likely a promising therapeutic or preventive approach for NAFLD.

Acknowledgements We thank Dr. Lei Jiao from the Panovue Biological Technology Company (Beijing) for technical advice and Prof. Yunjun Zhang from the Peking University Health Science Center for statistical consultation. This work was supported by the National Natural Science Foundation of China (NSFC) (81870407), the National Key R\&D Program of China (2016YFE0116800), the China National Science and Technology Major Project for Infectious Diseases Control during the 13th Five-Year Plan Period (2017ZX10202202-005-002, 2018ZX09201002-001-005) and the Peking University People's Hospital Research and Development Fund (RDX2018-06).

\section{Compliance with ethical standards}

Conflict of interest The authors declare that they have no conflict of interest.
Publisher's note Springer Nature remains neutral with regard to jurisdictional claims in published maps and institutional affiliations.

\section{References}

1. Friedman SL, Neuschwander-Tetri BA, Rinella M, Sanyal AJ. Mechanisms of NAFLD development and therapeutic strategies. Nat Med. 2018;24:908-22.

2. Rotman Y, Sanyal AJ. Current and upcoming pharmacotherapy for non-alcoholic fatty liver disease. Gut. 2017;66:180-90.

3. Younossi ZM, Koenig AB, Abdelatif D, Fazel Y, Henry L, Wymer M. Global epidemiology of nonalcoholic fatty liver disease-Meta-analytic assessment of prevalence, incidence, and outcomes. Hepatology. 2016;64:73-84.

4. Younossi Z, Anstee QM, Marietti M, Hardy T, Henry L, Eslam M, et al. Global burden of NAFLD and NASH: trends, predictions, risk factors and prevention. Nat Rev Gastroenterol Hepatol. 2018;15:11-20.

5. Dulai PS, Singh S, Patel J, Soni M, Prokop LJ, Younossi Z, et al. Increased risk of mortality by fibrosis stage in nonalcoholic fatty liver disease: systematic review and meta-analysis. Hepatology. 2017;65:1557-65.

6. Estes C, Anstee QM, Arias-Loste MT, Bantel H, Bellentani S, Caballeria $\mathrm{J}$, et al. Modeling NAFLD disease burden in China, France, Germany, Italy, Japan, Spain, United Kingdom, and United States for the period 2016-2030. J Hepatol. 2018;69:896-904.

7. Lebeaupin C, Vallée D, Hazari Y, Hetz C, Chevet E, BaillyMaitre B. Endoplasmic reticulum stress signaling and the pathogenesis of non-alcoholic fatty liver disease. J Hepatol. 2018;69:927-47.

8. Ishikawa H, Barber GN. STING is an endoplasmic reticulum adaptor that facilitates innate immune signaling. Nature. 2008;455:674-8.

9. Ma F, Li B, Yu Y, Iyer SS, Sun M, Cheng G. Positive feedback regulation of type I interferon by the interferon-stimulated gene STING. EMBO Rep. 2015;16:202-12.

10. Iracheta-Vellve A, Petrasek J, Gyongyosi B, Satishchandran A, Lowe P, Kodys K, et al. Endoplasmic reticulum stress-induced hepatocellular death pathways mediate liver injury and fibrosis via stimulator of interferon genes. J Biol Chem. 2016;291:26794-805.

11. Petrasek J, Iracheta-Vellve A, Csak T, Satishchandran A, Kodys K, Kurt-Jones EA, et al. STING-IRF3 pathway links endoplasmic reticulum stress with hepatocyte apoptosis in early alcoholic liver disease. Proc Natl Acad Sci USA. 2013;110:16544-9.

12. Qiao JT, Cui C, Qing L, Wang LS, He TY, Yan F, et al. Activation of the STING-IRF3 pathway promotes hepatocyte inflammation, apoptosis and induces metabolic disorders in nonalcoholic fatty liver disease. Metabolism. 2018;81:13-24.

13. Lei Z, Deng M, Yi Z, Sun Q, Shapiro RA, Xu H, et al. cGASmediated autophagy protects the liver from ischemia/reperfusion injury independent of STING. Am J Physiol Gastrointest Liver Physiol. 2018;314:G655-G667.

14. Thomsen MK, Nandakumar R, Stadler D, Malo A, Valls RM, Wang F, et al. Lack of immunological DNA sensing in hepatocytes facilitates hepatitis $\mathrm{B}$ virus infection. Hepatology. 2016;64:746-59.

15. Luo X, Li H, Ma L, Zhou J, Guo X, Woo SL, et al. Expression of STING is increased in liver tissues from patients with NAFLD and promotes macrophage-mediated hepatic inflammation and fibrosis in mice. Gastroenterology. 2018;155:1971-84.

16. Yu Y, Liu Y, An W, Song J, Zhang Y, Zhao X. STING-mediated inflammation in Kupffer cells contributes to progression of nonalcoholic steatohepatitis. J Clin Invest. 2019;129:546-55. 
17. Tacke F, Zimmermann HW. Macrophage heterogeneity in liver injury and fibrosis. J Hepatol. 2014;60:1090-6.

18. Krenkel O, Tacke F. Liver macrophages in tissue homeostasis and disease. Nat Rev Immunol. 2017;17:306-21.

19. Tacke F. Targeting hepatic macrophages to treat liver diseases. J Hepatol. 2017;66:1300-12.

20. Krenkel O, Mossanen JC, Tacke F. Immune mechanisms in acetaminophen-induced acute liver failure. Hepatobiliary Surg Nutr. 2014;3:331-43.

21. Krenkel O, Puengel T, Govaere O, Abdallah AT, Mossanen JC, Kohlhepp M, et al. Therapeutic inhibition of inflammatory monocyte recruitment reduces steatohepatitis and liver fibrosis. Hepatology. 2018;67:1270-83.

22. Kazankov K, Barrera F, Moller HJ, Rosso C, Bugianesi E, David $\mathrm{E}$, et al. The macrophage activation marker sCD163 is associated with morphological disease stages in patients with non-alcoholic fatty liver disease. Liver Int. 2016;36:1549-57.

23. Navarro LA, Wree A, Povero D, Berk MP, Eguchi A, Ghosh S, et al. Arginase 2 deficiency results in spontaneous steatohepatitis: a novel link between innate immune activation and hepatic de novo lipogenesis. J Hepatol. 2015;62:412-20.

24. Wehr A, Baeck C, Heymann F, Niemietz PM, Hammerich L, Martin C, et al. Chemokine receptor CXCR6- dependent hepatic NK T Cell accumulation promotes inflammation and liver fibrosis. J Immunol. 2013;190:5226-36.

25. Ehling J, Bartneck M, Wei X, Gremse F, Fech V, Möckel D, et al. CCL2-dependent infiltrating macrophages promote angiogenesis in progressive liver fibrosis. Gut. 2014;63:1960-71.

26. Ju C, Tacke F. Hepatic macrophages in homeostasis and liver diseases: from pathogenesis to novel therapeutic strategies. Cell Mol Immunol. 2016;13:316-27.

27. Brunt EM, Janney CG, Di Bisceglie AM, Neuschwander-Tetri BA, Bacon BR. Nonalcoholic steatohepatitis: a proposal for grading and staging the histological lesions. Am J Gastroenterol. 1999;94:2467-74.

28. Kleiner DE, Brunt EM, Van Natta M, Behling C, Contos MJ, Cummings OW, et al. Design and validation of a histological scoring system for nonalcoholic fatty liver disease. Hepatology. 2005;41:1313-21.

29. Kleiner DE, Makhlouf HR. Histology of nonalcoholic fatty liver disease and nonalcoholic steatohepatitis in adults and children. Clin Liver Dis. 2016;20:293-312.

30. Mao Y, Luo W, Zhang L, Wu W, Yuan L, Xu H, et al. STINGIRF3 triggers endothelial inflammation in response to free fatty acid-induced mitochrondrial damage in diet-induced obesity. Arterioscler Thromb Vasc Biol. 2017;37:920-9.

31. Alisi A, Carpino G, Oliveira FL, Panera N, Nobili V, Gaudio E. The role of tissue macrophage-mediated inflammation on NAFLD pathogenesis and its clinical implications. Mediators Inflamm. 2017;2017:8162421.

32. Baeck C, Wehr A, Karlmark KR, Heymann F, Vucur M, Gassler $\mathrm{N}$, et al. Pharmacological inhibition of the chemokine CCL2 (MCP-1) diminishes liver macrophage infiltration and steatohepatitis in chronic hepatic injury. Gut. 2012;61:416-26.

33. Mcguinness PH, Painter D, Davies S, McCaughan GW. Increases in intrahepatic CD68 positive cells, MAC387 positive cells, and proinflammatory cytokines (particularly interleukin 18 ) in chronic hepatitis C infection. Gut. 2000;46:260-9.

34. Strauss O, Dunbar PR, Bartlett A, Phillips A. The immunophenotype of antigen presenting cells of the mononuclear phagocyte system in normal human liver-a systematic review. J Hepatol. 2015;62:458-68.

35. Antoniades CG, Quaglia A, Taams LS, Mitry RR, Hussain M, Abeles R, et al. Source and characterization of hepatic macrophages in acetaminophen induced acute liver failure in humans. Hepatology. 2012;56:735-46.

36. Mossanen JC, Krenkel O, Ergen C, Govaere O, Liepelt A, Puengel $\mathrm{T}$, et al. Chemokine (C-C motif) receptor 2-positive monocytes aggravate the early phase of acetaminophen-induced acute liver injury. Hepatology. 2016;64:1667-82.

37. Subimerb C, Pinlaor S, Lulitanond V, Khuntikeo N, Okada S, McGrath MS, et al. Circulating CD14(+) CD16(+) monocyte levels predict tissue invasive character of cholangiocarcinoma. Clin Exp Immunol. 2010;161:471-9.

38. du Plessis J, van Pelt J, Korf H, Mathieu C, van der Schueren B, Lannoo M, et al. Association of adipose tissue inflammation with histologic severity of nonalcoholic fatty liver disease. Gastroenterology. 2015;149:635-48.

39. Gadd VL, Skoien R, Powell EE, Fagan KJ, Winterford C, Horsfall $\mathrm{L}$, et al. The portal inflammatory infiltrate and ductular reaction in human nonalcoholic fatty liver disease. Hepatology. 2014;59:1393-405.

40. Gonzalez-Dominguez E, Samaniego R, Flores-Sevilla JL, CamposCampos SF, Gómez-Campos G, Salas A, et al. CD163L1 and CLEC5A discriminate subsets of human resident and inflammatory macrophages in vivo. J Leukoc Biol. 2015;98:453-66.

41. Antoniades CG, Khamri W, Abeles RD, Taams LS, Triantafyllou E, Possamai LA, et al. Secretory leukocyte protease inhibitor: a pivotal mediator of anti-inflammatory responses in acetaminophen induced acute liver failure. Hepatology. 2014;59:1564-76.

42. Song E, Ouyang N, Hörbelt M, Antus B, Wang M, Exton MS. Influence of alternatively and classically activated macrophages on fibrogenic activities of human fibroblasts. Cell Immunol. 2000;204:19-28.

43. Karlmark KR, Weiskirchen R, Zimmermann HW, Gassler N, Ginhoux F, Weber C, et al. Hepatic recruitment of the inflammatory Gr1+ monocyte subset upon liver injury promotes hepatic fibrosis. Hepatology. 2009;50:261-74.

44. Liu S, Cai X, Wu J, Cong Q, Chen X, Li T, et al. Phosphorylation of innate immune adaptor proteins MAVS, STING, and TRIF induces IRF3 activation. Science. 2015;347:aaa2630.

45. Barber GN. STING-dependent cytosolic DNA sensing pathways. Trends Immunol. 2014;35:88-93.

46. Cho CS, Park HW, Ho A, Semple IA, Kim B, Jang I, et al. Lipotoxicity induces hepatic protein inclusions through TANK binding kinase 1-medicated p62/sequestosome 1 phosphorylation. Hepatology. 2018;68:1331-46.

47. Krapp C, Jønsson K, Jakobsen MR. STING dependent sensing-does HIV actually care? Cytokine Growth Factor Rev. 2018;40:68-76.

48. People's Republic of China health industry standards, WS/T 4282013. 